University of Nebraska - Lincoln

DigitalCommons@University of Nebraska - Lincoln

Civil and Environmental Engineering Faculty

Publications

Civil and Environmental Engineering

Fall 2016

\title{
A Primer on Vehicle-to-Barrier Communications: Effects of Roadside Barriers, Encroachment, and Vehicle Braking
}

\author{
Samil Temel \\ Turkish Air Force, Izmir, Turkey, stemel@unl.edu \\ Mehmet C. Vuran \\ University of Nebraska-Lincoln, mcvuran@cse.unl.edu \\ Ronald K. Faller \\ University of Nebraska - Lincoln, rfaller1@unl.edu
}

Follow this and additional works at: https://digitalcommons.unl.edu/civilengfacpub

Temel, Samil; Vuran, Mehmet C.; and Faller, Ronald K., "A Primer on Vehicle-to-Barrier Communications: Effects of Roadside Barriers, Encroachment, and Vehicle Braking" (2016). Civil and Environmental Engineering Faculty Publications. 184.

https://digitalcommons.unl.edu/civilengfacpub/184

This Article is brought to you for free and open access by the Civil and Environmental Engineering at DigitalCommons@University of Nebraska - Lincoln. It has been accepted for inclusion in Civil and Environmental Engineering Faculty Publications by an authorized administrator of DigitalCommons@University of Nebraska Lincoln. 


\title{
A Primer on Vehicle-to-Barrier Communications: Effects of Roadside Barriers, Encroachment, and Vehicle Braking
}

\author{
Samil Temel* Mehmet C. Vuran ${ }^{\ddagger} \quad$ Ronald K. Faller ${ }^{\dagger}$ \\ ${ }^{*}$ Turkish Air Force NCO College, 35415, Gaziemir/Izmir, Turkey \\ $\ddagger$ Department of Computer Science and Engineering, ${ }^{\dagger}$ Midwest Roadside Safety Facility, \\ $\ddagger_{\dagger}$ University of Nebraska-Lincoln, Lincoln, Nebraska 68588 \\ Email: stemel@unl.edu,mcvuran@cse.unl.edu,rfaller1@unl.edu
}

\begin{abstract}
Today, more than half of the traffic fatalities are a result of run-off-road (RoR) crashes, which usually involve a single vehicle. Roadside barriers are often the last means to mitigate the severity of a RoR crash into hazardous objects or features. While the recent research on vehicular communications primarily focus on safety related wireless communications for vehicle-to-vehicle (V2V) and vehicle-to-infrastructure (V2I) scenarios, the interactions between vehicles and barriers in nextgeneration vehicular systems have not been well-studied.

In this paper, vehicle-to-barrier (V2B) wireless communication paradigm is introduced as a potential missing link in preventing single-vehicle RoR fatalities ${ }^{1}$. V2B communications, which take place between vehicles and radios embedded in roadside barriers can contribute to keeping cars on the road and help mitigate RoR crashes. The realization of V2B communication services necessitates an in-depth understanding of the underlying physical characteristics of the environment and channel. To this end, in this paper, some of the first real world field test measurement results of V2B communications are presented. More specifically, the effects of two types of commonly-utilized barriers (rigid concrete barrier and corrugated-beam guardrail) on the V2B channel communications are illustrated. The results show that guardrail barriers exhibit a waveguiding effect on signal transmission, while higher signal attenuation is observed with rigid barriers. Moreover, experiments illustrate the characteristics of V2B orthogonal frequency-division multiplexing (OFDM) communication during vehicle encroachment and braking in terms of received signal strength, error vector magnitude, and phase error statistics. The results highlight that barrier-height antenna deployments result in high channel quality for long distances and are not influenced by mobility and vehicle brake during encroachment scenarios, making them a strong candidate for V2B communications.
\end{abstract}

\section{INTRODUCTION}

In the United States, traffic crashes kill nearly four people every hour [1]. More specifically, in 2014, 54\% of the traffic fatalities (17,791 fatalities) were a result of run-off-road (RoR) crashes. A RoR crash is defined as a crash which occurs after a vehicle crosses an edge line or a center line, or otherwise leaves the traveled way and collide into a fixed object such as a tree, rock, wall, barrier, or a median. [2].

${ }^{1}$ This work was conducted while $\mathrm{S}$. Temel was at the University of Nebraska-Lincoln and was partly supported by NSF CNS-0953900, CNS1247941, DBI-1331895, and CNS-1423379 awards.

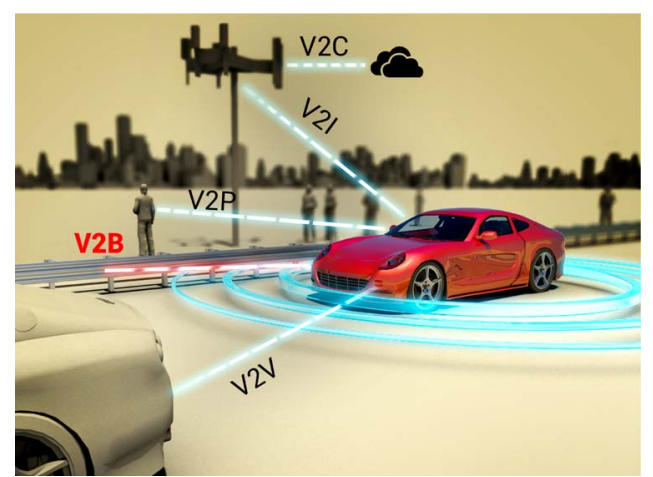

Fig. 1: Vehicular communication technologies.

The societal and economical consequences of traffic accidents have urged the academic and industry communities to design more efficient infrastructures. To this end, new technologies for connected vehicles, dubbed V2X; including vehicle-to-vehicle (V2V) [3], vehicle-to-infrastructure (V2I) [4], vehicle-to-pedestrian (V2P) [5], and vehicle-tocloud (V2C) [6] paradigms (Fig. 1); have been developed. Yet, connected vehicles of tomorrow and autonomous vehicles of the near future are slated to operate on roadside infrastructure, which was designed decades ago. In addition, recent sensory technologies such as blind-spot detection, lanedeparture warning, or adaptive cruise control, are still limited in preventing RoR fatalities. As such, new technologies for connected vehicles may not address safety issues related to single-vehicle RoR fatalities.

Considering the projected plans to provide wireless communication capabilities to each car, we believe it is time to bring roadside infrastructure technology (e.g., lane markings, barriers) into the V2X paradigm. To this end, we introduce the vehicle-to-barrier (V2B) communication paradigm. V2B communications will provide connectivity between vehicles and roadside barriers to help keep vehicles safely on the road (e.g., Fig. 2), complement on-board sensor technologies, avoid RoR crashes in advance, or minimize the severity of a crash if it is inevitable. V2B systems can augment physical barrier structures to greatly reduce the number of roadside encroachments, or even serve as a first line of defense (i.e., 


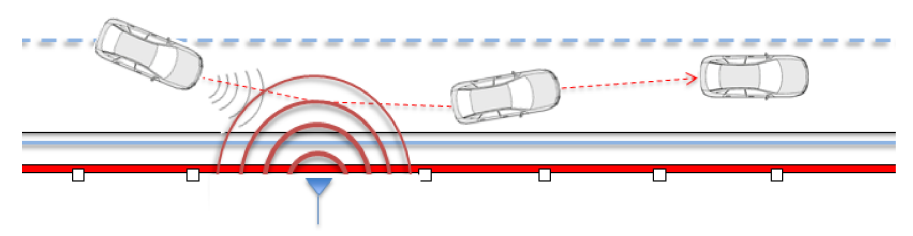

Fig. 2: V2B systems will help keep vehicles on road.

smart barrier systems). However, the realization of novel V2B communication services necessitates a deep understanding of the underlying physical characteristics of the environment and V2B channel.

To improve and promote safety, robustness, cooperation, and coordination between vehicles and roadside infrastructure, Dedicated Short Range Communication (DSRC) and IEEE 802.11p standards have recently been defined [7], [8]. Accordingly, orthogonal frequency-division multiplexing (OFDM) has become the de-facto communication technique. OFDM has been studied for most of the V2X systems [9]. However, V2B OFDM communication performance has not been studied. As such, the effects of barriers on wireless signals and behavior of OFDM transmission during vehicle encroachment and breaking should be evaluated to develop novel V2B communication techniques. In this study, we present the first real-world field test results conducted at the Midwest Roadside Safety Facility (MwRSF) and the University of Nebraska Lincoln (UNL) campus.

The remainder of this paper is organized as follows: In Section II, V2B communication paradigm is introduced. In Section III, related work is presented. In Section IV, test campaign setups are described, and in Section V, field test results and the corresponding discussions are presented. We conclude the paper is in Section VI.

\section{Vehicle to BARRIER (V2B) COMMUNiCATIONS}

To minimize the severity caused by RoR crashes, transportation researchers have been encouraged to develop effective roadside barriers to reduce the potential for overturns of the vehicles, eliminate crashes with fixed-object hazards, and decrease the social costs associated with the traffic accidents [10], [11]. Owing to the rapid advances in wireless communication technologies, we envision that V2B communication systems can be deployed along the roads to decrease the number and severity of passenger vehicle crashes. Providing a V2B communication between errant vehicles and barriers can potentially lead to rapid-response safety systems to detect an in-coming crash, alert the driver, and take necessary precautions. Although futuristic, we believe that the rapid decreases in the costs of wireless embedded systems and developments in renewable energy systems will help realize V2B systems. Accordingly, in the near future, V2B systems will augment physical barriers or will be deployed alone where it is costly or impossible to erect a barrier. Additionally, V2B systems would give rise to various safety-related applications as follows:
Vehicle-to-Barrier Crash Safety: V2B systems would help minimize the fatality of crashes by employing novel communication techniques between the barrier and vehicle for encroachment, crash, and post-crash phases.

Sustainable Safe Road Design: To achieve sustainable safe roads, the interaction between motorists, vehicles, and roadside barriers will have a major role. V2B communication systems will constantly provide information to authorities about the behavior of motorists, effect of the road conditions, which will enable them to develop safer roads and roadside barriers.

For the realization of these future applications, there is a need for field test measurements, data collection on vehicle encroachment and braking scenarios, communication experiments with crash tests, design and development of V2B communication protocols, and evaluations in simulated and emulated crash scenarios. To this end, the physical structure and characteristics of the errant vehicles exhibit the following challenges:

Fading and Doppler shift: The unpredictable movement behavior of the vehicles (especially during a crash) and fading characteristics of the roadside barriers, which have not been studied before, make it a challenging task to determine the fast fading effects.

Frequency offset: During a crash, the velocity of a vehicle drops drastically in a very short period of time (i.e., within 0.5 seconds). This phenomenon would lead to a dynamic Doppler shift behavior which could result in a mismatch between the oscillators of the transmitter and receivers.

Subcarrier determination: The determination of the number of OFDM subcarriers, pilot carriers, modulation methods, and cylic prefix length have to be tested in real world field tests.

Antenna specifications: Antenna type and deployment strategies have to be re-visited for V2B scenarios.

In this paper, we investigate the effects of barrier type on channel frequency response and effects of encroachment and brake on OFDM subcarriers. In addition, we present various real-world test results which are conducted with different antenna deployment strategies.

\section{RELATED WORK}

For nearly two decades, intelligent transportation systems (ITS) have been developed to provide transportation systems with information and communication facilities [12]. Since then, many vehicular communication systems have been successfully developed with V2X technologies. However, to the best of our knowledge, wireless communication between vehicles and barriers have not been considered yet.

The main critical aspect to be considered when deploying any wireless vehicular system is the influence of the underlying wireless technology. For V2V communications, DSRC has become the foremost communication stack owing to its performance over high speeds [8]. Besides, it has been shown for V2I that the Wireless Access in Vehicular Environments (WAVE) systems scale well and provide ample capacity as compared to digital broadcasting and cellular communication systems [13]. 
TABLE I: List of Equipment.

\begin{tabular}{|c||c||c||}
\hline Device & Description & Quantity \\
\hline VNA & Agilent Fieldfox N9923A & 1 \\
Cable & 4-m co-axial & 2 \\
Antenna & AIR802 Dual Band 5.3 dBi & 2 \\
USRP & B210 with USB 3.0 & 2 \\
USRP & E312 & 1 \\
Camera & ELP 2.1 mm Lens & 1 \\
\hline
\end{tabular}

For V2B communications, antenna deployment strategies (antenna type and height) on the roadside barriers have to be determined. For V2I applications, antenna heights of 3$15 \mathrm{~m}$ are considered to maintain a strong line-of-sight (LOS) with the vehicles [14] and effectively disseminate broadcast messages. Obstructions blocking the LOS significantly attenuate the signal and reduce the communication range, and static obstacles must not be neglected when designing V2I systems [15]. In V2I, the infrastructure plays a coordination role by gathering global or local information on traffic and road conditions and then, broadcasting it from high antenna towers to a group of vehicles [14], [16]. Communication efficiency can be greatly enhanced by placing road-side unit (RSU) antennas above all driving vehicles [17], and the rooftop is considered to be the optimum location for the onboard unit (OBU) antenna. It is also argued that low-height RSU antennas necessitate the usage of longer packet lengths, which results in increased signal-to-noise ratio (SNR) demands during transmission. In this context, when designing V2B technologies, antenna deployment is a crucial factor to analyze considering the relatively lower heights of existing barriers.

These studies mainly focus V2I scenarios on highway and urban areas, but the impacts of roadside barriers on wireless propagation is not captured. To this end, we analyze the effects of roadside barriers on wireless signal propagation to cast light on the future V2B communication technologies.

\section{FIELD EXPERIMENTS}

To evaluate the effects of barriers on V2B communications, we conducted two measurement campaigns at the MwRSF outdoor proving grounds and UNL campus. Both test sites were prohibited to public traffic. First, we investigate the effects of the barrier type on wireless signal transmission by evaluating the impulse response of the channel. Second, experiments with a vehicle and a barrier setup were conducted to study the impacts of encroachment and vehicle braking on OFDM symbols. The equipment for the two campaigns are listed in Table I. Next, we present the experiment setups, and the results are presented in Section V.

\section{A. Barrier Type}

As stated in Section II, V2B systems will mainly reside on the barriers alongside the roads. Hence, understanding the effects of barrier structures on wireless communication signals is essential for V2B system development. In these field tests, we conducted experiments with two types of commonly-utilized systems: corrugated-beam guardrail and rigid concrete barriers. In addition, as benchmark results, we

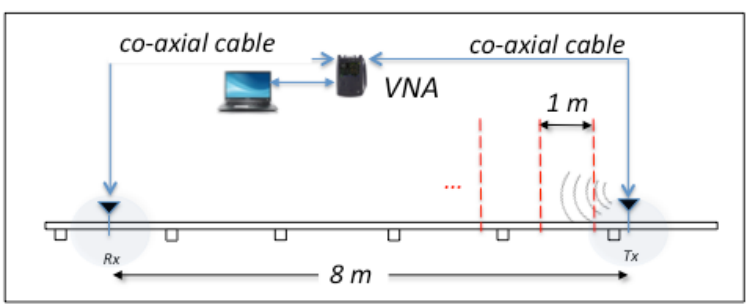

Fig. 3: Measurement setup for barrier type experiments.

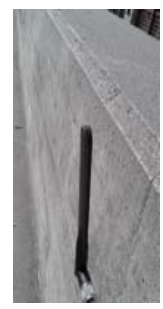

(a) Rx antenna

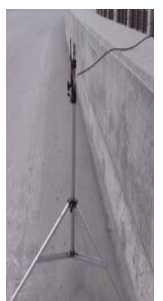

(b) Tx antenna

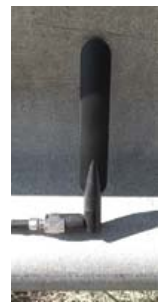

(c) Rx antenna

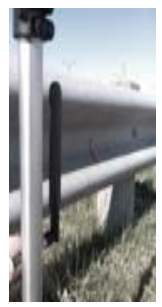

(d) Tx antenna
Fig. 4: Antenna placement for (a-b) rigid concrete barrier and (c-d) corrugated-beam guardrail.

conducted measurements in open space with the same ground conditions.

The channel impulse response and corresponding path loss measurements are obtained from frequency domain measurements of the channel using a vector network analyzer (VNA). VNA generated a linearly swept frequency signal [18] over a frequency range of $2 \mathrm{GHz}$ to $3 \mathrm{GHz}$ with 200 frequency bins. The measurements were collected using Standard Commands for Programmable Instruments (SCPI) scripts at a laptop computer. Two $4 \mathrm{~m}$ co-axial cables with transmitter (Tx) and receiver $(\mathrm{Rx})$ antennas were attached to two ports of the VNA, as shown in Fig. 3. The transmission power was set to $5 \mathrm{dBm}$, which is the maximum for the VNA. At every $1 \mathrm{~m}$ distance, we took $10 \mathrm{~S} 21$ measurements and averaged the results. This setup has two limitations: measurements are limited to the 3 $\mathrm{GHz}$ band due to the device limitations and cannot capture the $5.9 \mathrm{GHz}$ band. Moreover, distances are limited to only $8 \mathrm{~m}$ due to cable limitations. Yet, the comparative approach used in the experiments provides valuable results for barrier type impacts despite the limitations.

In all experiments, the Rx antenna was fixed and the $\mathrm{Tx}$ antenna was moved at $1 \mathrm{~m}$ intervals up to a Tx-Rx distance of $8 \mathrm{~m}$. Impacts of the barriers were analyzed by conducting the measurements at different distances to the barrier. First, to establish a benchmark, measurements were taken in open space with the same ground properties (6 $\mathrm{m}$ from the barrier). For the rigid concrete barrier, the ground was concrete pavement and for the corrugated-beam guardrail, the ground was grassy with crush sand beneath.

For rigid concrete barrier experiments, Rx antenna was placed on the barrier and $2 \mathrm{~cm}$ away from the barrier surface (Fig. 4a) and Tx antenna was placed at barrier-Tx separations of $2 \mathrm{~cm}, 8 \mathrm{~cm}, 32 \mathrm{~cm}$, and $128 \mathrm{~cm}$ (Fig. 4b). The height and width of the concrete barrier were $81 \mathrm{~cm}$ and $40 \mathrm{~cm}$, 


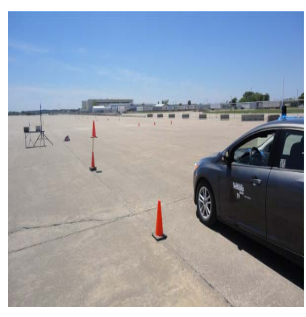

(a) Brake test setup

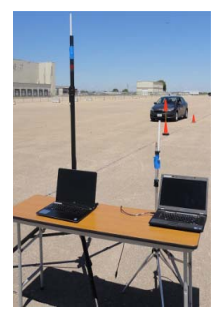

(b) Rx antennas

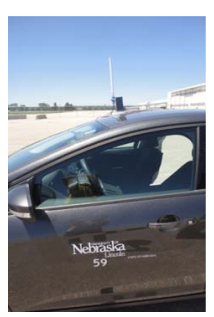

(c) Tx antenna
Fig. 5: $5.8 \mathrm{GHz}$ brake test setup.

respectively. For corrugated-beam guardrail, Rx antenna was placed in the trench of the $\mathrm{W}$-beam rail (Fig. 4c) and $\mathrm{Tx}$ antenna was placed at barrier-Tx separations of $2 \mathrm{~cm}, 8 \mathrm{~cm}$, $32 \mathrm{~cm}$, and $128 \mathrm{~cm}$ (Fig. 4d). The heights of the Tx and Rx antennas were $60 \mathrm{~cm}$.

\section{B. Vehicle Encroachment and Braking}

In this test campaign, we analyze the characteristics of OFDM transmissions during a vehicle encroachment, brake, and post-brake periods, which best mimic a car-crash scenario. During this test campaign, GNU Radio software development toolkit and USRP devices were used to transmit and receive OFDM symbols. A sequence of random bits were first parallelized and Inverse Fast Fourier Transform (IFFT) was applied to generate OFDM symbols. At the receiver side, the received signal stream (IQ data) was first parallelized, and a reverse FFT process was applied to generate the received symbols. Experiments were conducted at $5.8 \mathrm{GHz}$ and $2.4 \mathrm{GHz}$ bands.

1) Brake Tests at $5.8 \mathrm{GHz}$ : For the tests, a Toyota sedan car was used with an encroachment velocity of $40 \mathrm{mph}$ with the help of cruise control in open space experiments (Figs. 5). A full brake was applied when the vehicle was $10 \mathrm{~m}$ away from the receiver. An omni-directional antenna was attached on the roof-top of the vehicle $(1.4 \mathrm{~m})$, which was connected to the transmitter USRP (E312) with a coaxial cable (8 dB return loss). During the tests, we installed two omni-directional antennas to two receiver USRPs (B210) with the same type of coaxial cables. We studied four different receiver antenna heights of $0.82 \mathrm{~m}, 1.4 \mathrm{~m}, 1.9 \mathrm{~m}$, and $3 \mathrm{~m}$, which represent the heights of a concrete barrier, rooftop of a sedan vehicle, rooftop of a SUV vehicle, and a traffic signal, respectively. The center frequency was set to $5,800 \mathrm{MHz}$. The sampling rate of the OFDM symbols was set to $500 \mathrm{kS} / \mathrm{sec}^{2}$ with a packet length of 128 bytes, BPSK modulation, and FFT length of 64 with 48 occupied subcarriers, which mostly reflect the DSRC physical layer parameters [14].

2) Brake Tests at $2.4 \mathrm{GHz}$ : For the tests, a Ford sedan car, with a roof-top height of $1.4 \mathrm{~m}$, was used. The test scenario is shown in Fig. 6, where the vehicle was moved toward the roadside barrier at an angle of 30 degrees with an encroachment velocity of $30 \mathrm{mph}$. A full brake was applied when the vehicle was $5 \mathrm{~m}$ away from the receiver.

\footnotetext{
${ }^{2}$ DSRC uses a higher bandwidth, where higher sampling rates were not possible due to equipment limitations.
}

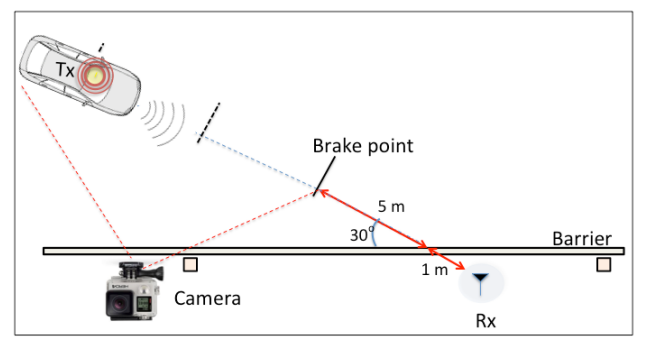

Fig. 6: $2.4 \mathrm{GHz}$ brake test scenario.

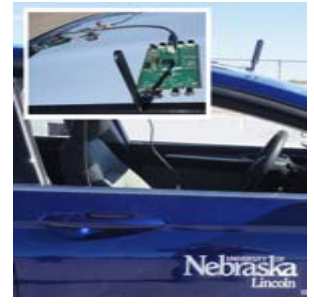

(a) Tx USRP on the roof-top

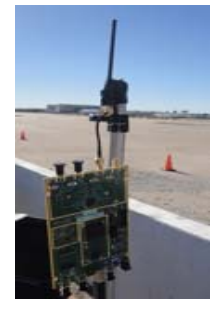

(b) Rx USRP behind the barrier
Fig. 7: $2.4 \mathrm{GHz}$ brake test setup.

The longitudinal acceleration history of the vehicle is logged to determine the period of the brake. Before the tests, the encroachment lane was marked with cones at $5 \mathrm{~m}$ intervals and the correspondent OFDM symbols to each distance and brake time is determined by analyzing the recorded video files frameby-frame. First, we conducted tests while the Rx antenna was installed behind a rigid concrete barrier (1.4 m height) and on the barrier $(0.82 \mathrm{~m})$ in MwRSF fields. Then, tests were repeated in open space (for both $1.4 \mathrm{~m}$ and $0.82 \mathrm{~m}$ antenna heights) with the same ground properties to analyze the barrier impact in similar encroachment conditions.

As shown in Fig. 7, the Tx USRP was attached on the roof-top of the vehicle, which was connected to a laptop inside the vehicle with a USB cable. The receiver USRP was installed $1 \mathrm{~m}$ behind the roadside barrier. In advance of the tests, all of the laptop computers are time synchronized over Internet. During the tests, the center frequency was set to 2,430 MHz with an FFT length of 256 with 192 occupied subcarriers. All other parameters were the same as $5.8 \mathrm{GHz}$ experiments. Throughout the tests, all the packet reception steps, encroachment video, and acceleration were logged for post-processing.

\section{RESUlts AND Discussions}

\section{A. Barrier Type}

Average path loss as a function of distance for the concrete barrier is shown in Fig. 8a. Compared to the open space results, path loss is 8.7 - $14.8 \mathrm{~dB}$ higher depending on the separation distance. Moreover, greater barrier-Tx separation results in a lower path loss. This clearly reflects the attenuating effect of the concrete barrier especially when the antennas are deployed close to the barrier.

When deployed on a guardrail, V2B path loss decreases significantly, as shown in Fig. 8b. Compared to the concrete 


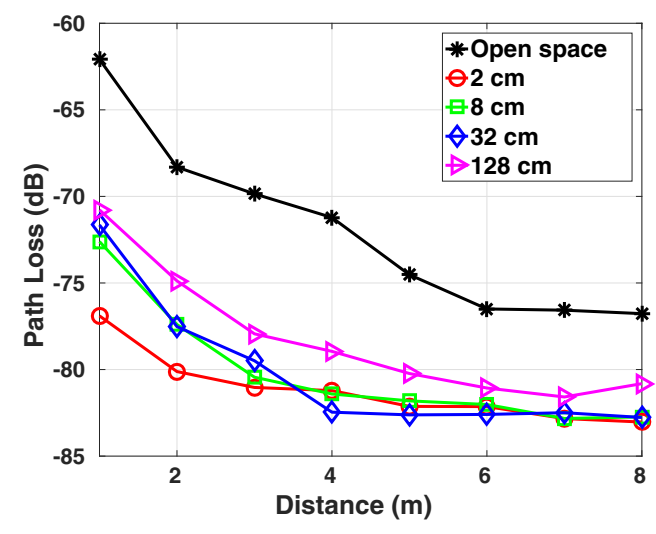

(a) Rigid Concrete Barrier

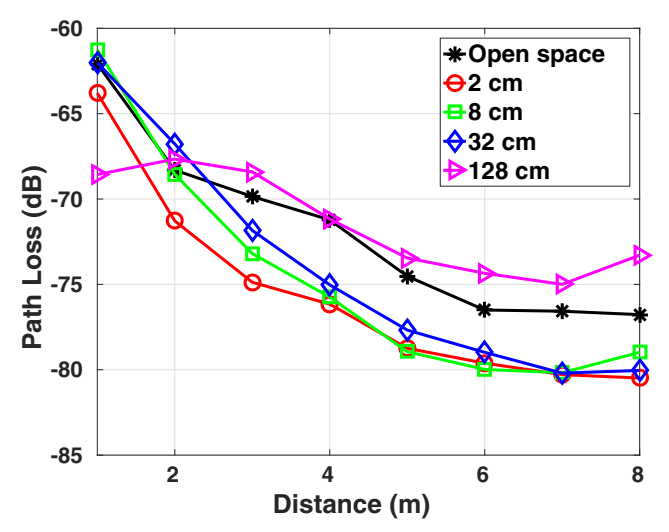

(b) Corrugated-beam Guardrail

Fig. 8: Path loss for different barrier-Tx antenna separations.

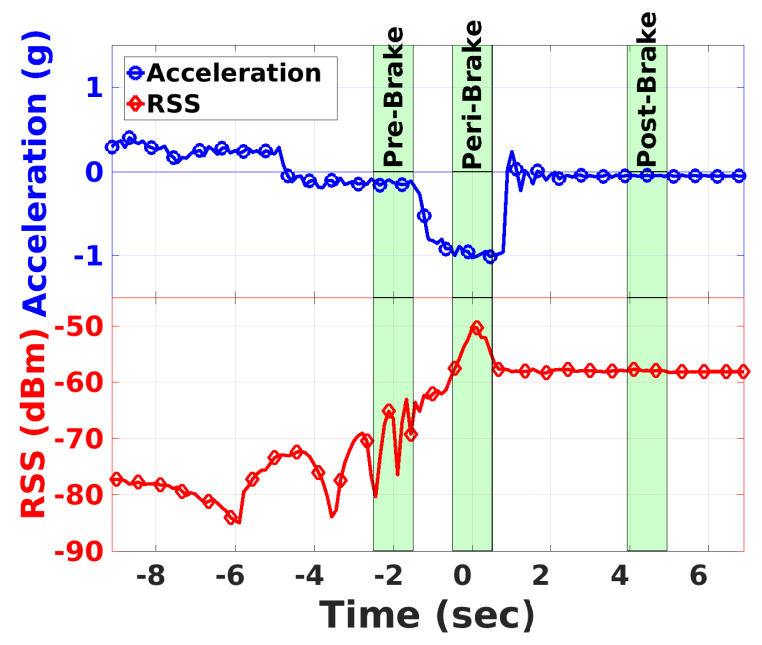

Fig. 9: Accelerometer and received signal strength (RSS) during a braking test.

barrier, guardrail has a 5.2 - $6.7 \mathrm{~dB}$ lower impact on path loss. Moreover, for a barrier-Tx separation of $128 \mathrm{~cm}$, guardrail improves signal strength by up to $6.5 \mathrm{~dB}$ compared to open space results. The results show the additive wave-guiding effect of the corrugated-beam guardrail that can be utilized for longer communication distances.

\section{B. Vehicle Encroachment and Braking}

To evaluate the OFDM impairments caused by vehicle speed (Doppler) and brake, we selected three intervals of 1s before, during, and after the brake as shown in Fig. 9. First, the middle of the brake duration, $t_{b}$, is estimated based on the accelerometer results. We also verified our braking time with the video files, where the sampling rate of the video files was $17.5 \mathrm{fps}$. Then, the intervals are defined as follows: Pre-brake $\left[t_{b}-2.5, t_{b}-1.5\right]$, peri-brake $\left[t_{b}-0.5, t_{b}+0.5\right]$, and postbrake $\left[t_{b}+4, t_{b}+5\right]^{3}$. The pre-brake interval is important for V2B communications because any crash avoidance-related communication may be conducted at this time. The peri-brake

\footnotetext{
${ }^{3}$ We use $t_{b}=0$ in the following for clarity.
}

interval includes a drastic change in acceleration and velocity as well as vibration due to the impacts of the brake. Finally, the post-brake interval is selected such that the vehicle is stationary and provides a good comparative metric to evaluate the Doppler effects.

We evaluate the received signal strength (RSS), error vector magnitude (EVM), and phase error (PE) of received OFDM symbols. RSS reflects the average received power of a single OFDM symbol. EVM is a measure of the deviation of the received symbol from the original transmitted data symbol on the IQ plane. Finally, PE is the angle of the received IQ data with respect to the ideal constellation point.

1) Brake Tests at $5.8 \mathrm{GHz}$ : For each of the four Rx antenna heights, the results are shown in Figs. 10 for the encroachment phase $(10-150 \mathrm{~m})$ as a function of Tx-Rx distance. It can be observed that barrier antenna height has a significant impact. The lowest deployment height of $0.82 \mathrm{~m}$ (typical barrier height) leads to a stable signal quality with distance (Fig. 10a). Moreover, EVM is decreased by up to $8.71 \mathrm{~dB}$ compared to other antenna heights (Fig. 10b). Signal quality and error statistics worsen with increasing antenna height. Abrupt changes in RSS, EVM, and PE for higher antenna heights can also be observed at distances 10-40m, which correspond to the time when cruise control was in effect ($5 \mathrm{~s}$ to $-2 \mathrm{~s}$ in Fig. 9). This illustrates the impacts of Doppler. Interestingly, $0.82 \mathrm{~m}$ deployment was minimally affected from these changes. Overall, the results suggest that antennas can be directly affixed to existing barriers for V2B communications without any height extensions.

In addition, for each of the three intervals, we evaluate the EVM and PE statistics of each subcarrier, as shown with cumulative distribution function (CDF) plots in Fig. 11 and Fig. 12, respectively. A summary of average EVM and PE results is also shown in Table II. It can be observed that the vehicle brake has a negative effect on wireless signal transmission, especially with high antenna heights. For example, the $3 \mathrm{~m}$ deployment results in EVMs of $-12.31 \mathrm{~dB},-12.55$ $\mathrm{dB}$, and $-17.31 \mathrm{~dB}$ on average, for pre-, peri-, and post-brake intervals, respectively. The $\sim 5 \mathrm{~dB}$ increase in EVM in pre- 


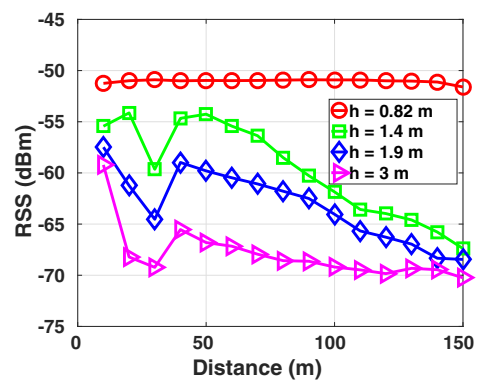

(a) RSS

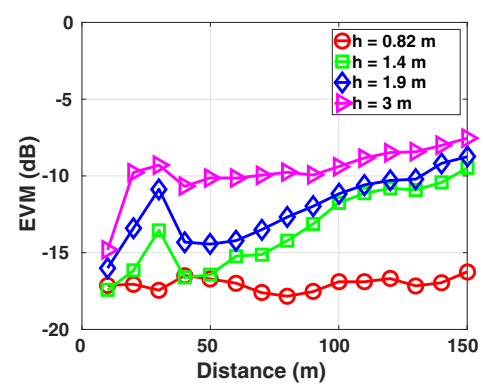

(b) EVM

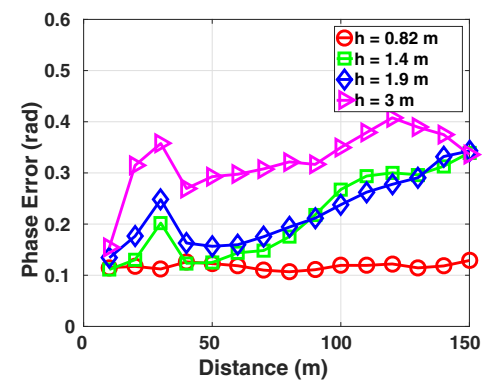

(c) PE

Fig. 10: OFDM signal transmission results for vehicle encroachment phase.

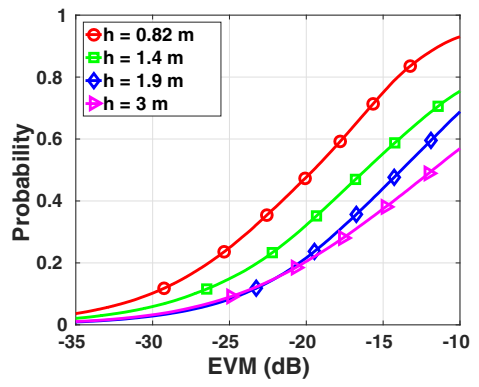

(a) Pre-brake

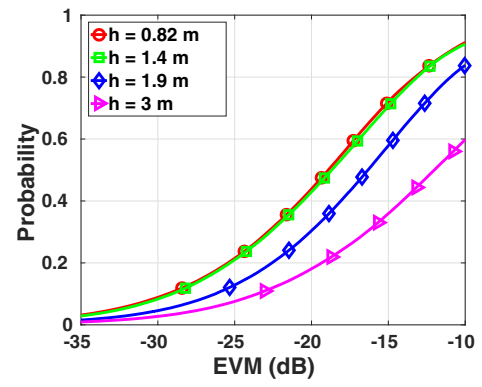

(b) Peri-brake

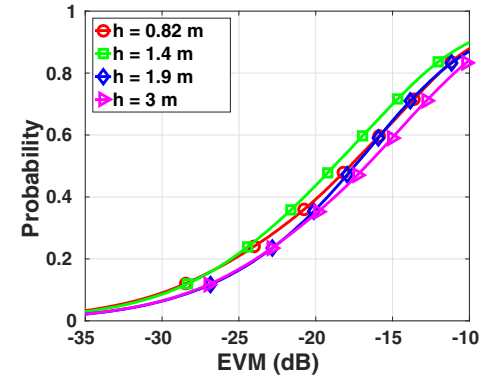

(c) Post-brake

Fig. 11: CDF of EVM results on occupied subcarriers.

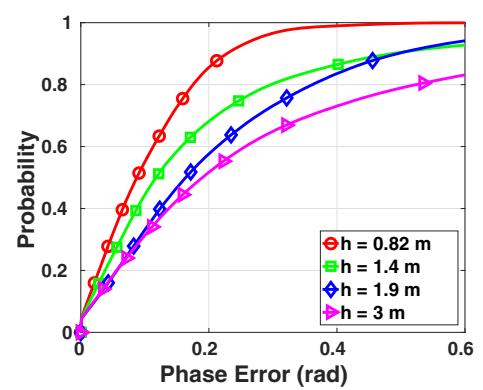

(a) Pre-brake

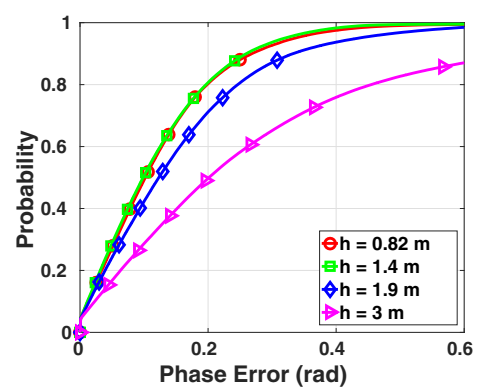

(b) Peri-brake

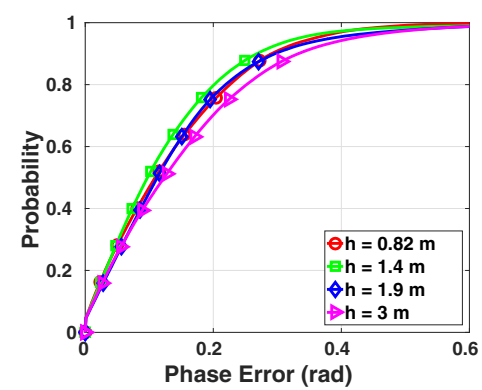

(c) Post-brake

Fig. 12: Phase error results on occupied subcarriers.

and peri-brake intervals compared to post-brake interval, when the vehicle was stationary, illustrates the impacts of mobility and Doppler. Similar observations can be made for $1.4 \mathrm{~m}$ and $1.9 \mathrm{~m}$ deployments. On the other hand, for the barrierheight $(0.82 \mathrm{~m})$ deployment, $0.82-1.52 \mathrm{~dB}$ improvement in EVM is observed while mobile. Similar observations can be made for phase errors in Fig. 12. While the deployment height is inconsequential for stationary communications (post-brake), barrier-height results in the lowest PE (0.1-0.13 rad) during encroachment and brake.

2) Brake Tests at $2.4 \mathrm{GHz}$ : For each of the three phases, we analyzed the signal impairments (EVM and PE) mainly caused by the barrier and vehicle brake. The average results are summarized in Table II and detailed results can be found in [19]. During a peri-break interval, Rx antenna on the barrier $(0.82 \mathrm{~m})$ results in nearly $3 \mathrm{~dB}$ increase in EVM compared to behind the barrier deployment $(1.4 \mathrm{~m})$. On average, barrier results in $4.46 \mathrm{~dB}, 9.94 \mathrm{~dB}$, and $2.49 \mathrm{~dB}$ decrease in EVM for $1.4 \mathrm{~m}$ barrier antenna in pre-, peri-, and post-break intervals, respectively. For deployments on the barrier, EVM increases by $2.2-2.6 \mathrm{~dB}$ in peri- and post-brake intervals, compared to open space experiments. In addition, the impacts of Doppler can be observed by comparing post-brake EVM and PE results with the higher values in pre- and peri-brake intervals.

3) Overview of the Test Results: According to the encroachment and brake tests conducted on $5.8 \mathrm{GHz}$ frequency band, we summarize the main outcomes of the results as follows:

For the encroachment phase, we observed a $15.53 \mathrm{~dB}$ improvement on signal strength when the receiver antennas are deployed on lower heights (i.e. $0.82 \mathrm{~m}$ - barrier height) than higher ones (i.e. $3 \mathrm{~m}$ - traffic signal height). Parallel to the received power results, we observed $15.52 \mathrm{~dB}$ better 
TABLE II: Summary of Vehicle Encroachment and Breaking Test Results.

\begin{tabular}{|c|c|c|c|c|c|c|c|c|c|}
\hline \multirow{3}{*}{ Metric } & \multirow{3}{*}{ Phase } & \multicolumn{4}{|c|}{$5.8 \mathrm{GHz}$} & \multicolumn{4}{|c|}{$2.4 \mathrm{GHz}$} \\
\hline & & \multirow{2}{*}{$\mathrm{h}=0.82 \mathrm{~m}$} & \multirow{2}{*}{$\mathrm{h}=0.1 .4 \mathrm{~m}$} & \multirow{2}{*}{$\mathrm{h}=1.9 \mathrm{~m}$} & \multirow{2}{*}{$\mathrm{h}=3 \mathrm{~m}$} & \multicolumn{2}{|c|}{$\mathrm{h}=0.82 \mathrm{~m}$} & \multicolumn{2}{|c|}{$\mathrm{h}=1.4 \mathrm{~m}$} \\
\hline & & & & & & Barrier & Open & Barrier & Open \\
\hline \multirow{3}{*}{ EVM $(\mathrm{dB})$} & Pre-brake & -19.98 & -16.01 & -14.14 & -12.31 & -13.34 & -12.98 & -12.91 & -8.45 \\
\hline & Peri-brake & -19.28 & -19.37 & -16.65 & -12.55 & -15.23 & -17.89 & -18.44 & -8.50 \\
\hline & Post-brake & -18.46 & -19.02 & -17.77 & -17.31 & -15.80 & -18.00 & -19.50 & -17.01 \\
\hline \multirow{3}{*}{ PE (rad) } & Pre-brake & 0.1075 & 0.2025 & 0.2226 & 0.3208 & 0.2291 & 0.2607 & 0.2520 & 0.3939 \\
\hline & Peri-brake & 0.1281 & 0.1236 & 0.1576 & 0.2919 & 0.1851 & 0.1320 & 0.1306 & 0.3935 \\
\hline & Post-brake & 0.1352 & 0.1280 & 0.1427 & 0.1557 & 0.1704 & 0.1304 & 0.1145 & 0.1541 \\
\hline
\end{tabular}

results in terms of EVM impairment results on lower heights, specifically for barrier height deployments.

For the brake intervals (pre-brake, peri-brake and postbrake), the $3 \mathrm{~m}$ receiver antenna deployment exhibits the worst results for signal impairments. In addition, we observe that lower barrier antenna heights result in lower errors and are not significantly affected by mobility or brake compared to higher heights.

For V2B networks, we mainly project deployment of antennas on the barriers and/or close to the height barriers. Hence, there is need to tailor V2B communication solutions to these heights and minimize the effects of signal impairments during critical encroachments to minimize fatalities.

\section{CONCLUSION}

Vehicle to barrier (V2B) communications a novel type of wireless technology which aim to achieve safer journeys for motorists by establishing safety-related communications between vehicles and roadside barriers. The main goals of V2B systems are to augment existing infrastructure to help keep passenger cars on road, exchange information, and potentially take the control of an errant vehicle to make crucial safety maneuvers when a run-off-road crash is inevitable. To assist potential future V2B deployments, real-world field test results are presented, which reveal the effects of barrier types on wireless communication. We mainly investigate two types of barriers: rigid concrete barrier and corrugated-beam guardrail. In addition, we present the main characteristics of OFDM symbols during a vehicle pre-brake, peri-brake, and post-brake intervals through vehicle encroachment and brake experiments. According to the test results, we observed more efficient signal results with corrugated-beam guardrail as compared to rigid concrete barriers. We also observed the adverse effect of braking on OFDM transmission in terms of received signal strength, error vector magnitude, and phase error results. Yet, barrier-height antenna deployments lead to low and sustained error rates, providing a motivation for the development of V2B communication systems.

\section{REFERENCES}

[1] U.S. Department of Transportation, "A compilation of motor vehicle crash data from the fatality analysis reporting system and the general estimates system," Traffic Safety Facts 2013, 2013.

[2] "U.S. Department of Transportation," http://safety.fhwa.dot.gov/roadway_dept/, accessed: 2016-02-28.

[3] A. F. Molisch, F. Tufvesson, J. Karedal, and C. F. Mecklenbrauker, "A survey on vehicle-to-vehicle propagation channels," IEEE Wireless Communications, vol. 16, no. 6, pp. 12-22, December 2009.
[4] T. Sukuvaara and P. Nurmi, "Wireless traffic service platform for combined vehicle-to-vehicle and vehicle-to-infrastructure communications," IEEE Wireless Communications, vol. 16, no. 6, pp. 54-61, 2009.

[5] N. Liu, M. Liu, J. Cao, G. Chen, and W. Lou, "When transportation meets communication: V2P over VANETs," in Distributed Computing Systems (ICDCS), 2010 IEEE 30th International Conference on, June 2010, pp. 567-576.

[6] R. Hussain, J. Son, H. Eun, S. Kim, and H. Oh, "Rethinking vehicular communications: Merging vanet with cloud computing," in Cloud Computing Technology and Science (CloudCom), 2012 IEEE 4th International Conference on, Dec 2012, pp. 606-609.

[7] V. D. Khairnar and K. Kotecha, "Performance of vehicle-to-vehicle communication using IEEE $802.11 \mathrm{p}$ in vehicular ad-hoc network environment," International Journal of Network Security, vol. 5, no. 2, pp. 143-170, March 2013.

[8] "IEEE standard for wireless access in vehicular environments (WAVE) network systems corrigendum 2: Miscellaneous corrections," IEEE Std 1609.3-2010/Cor 2-2014 (Corrigendum to IEEE Std 1609.3-2010), pp. 1-33, Dec 2014.

[9] C. Liu and Y. Bai, "Comparison of synchronization algorithms for ofdmbased vehicular ad hoc network," in Consumer Electronics, Communications and Networks (CECNet), 2012 2nd International Conference on, April 2012, pp. 942-945.

[10] T. F. H. Administration, "Fhwa roadway departure (rwd) strategic plan," FHWA Publications, 2013.

[11] R. Faller, K. Polivka, B. Kuipers, R. Bielenberg, J. Reid, J. Rohde, and D. Sicking, "Midwest guard-rail system for standard and special applications," Transportation Research Record: Journal of the Transportation Research Board, vol. 1890, no. 3, pp. 19-33, 2004.

[12] L. D. Baskar, B. D. Schutter, J. Hellendoorn, and Z. Papp, "Traffic control and intelligent vehicle highway systems: a survey," IET Intelligent Transport Systems, vol. 5, no. 1, pp. 38-52, March 2011.

[13] P. Belanovic, D. Valerio, A. Paier, T. Zemen, F. Ricciato, and C. F. Mecklenbrauker, "On wireless links for vehicle-to-infrastructure communications," IEEE Transactions on Vehicular Technology, vol. 59, no. 1, pp. 269-282, Jan 2010.

[14] J. Gozalvez, M. Sepulcre, and R. Bauza, "IEEE 802.11p vehicle to infrastructure communications in urban environments," IEEE Coтmunications Magazine, vol. 50, no. 5, pp. 176-183, May 2012.

[15] R. Meireles, M. Boban, P. Steenkiste, O. Tonguz, and J. Barros, "Experimental study on the impact of vehicular obstructions in vanets," in Vehicular Networking Conference (VNC), 2010 IEEE, Dec 2010, pp. 338-345.

[16] "U.S. Department of Transportation, Vehicle-toInfrastructure (V2I) Communications for Safety," http://www.its.dot.gov/safety/v2i_comm_safety.htm, accessed: 2016-0228.

[17] A. Paier, D. Faetani, and C. F. Mecklenbruker, "Performance evaluation of IEEE $802.11 \mathrm{p}$ physical layer infrastructure-to-vehicle real-world measurements," in Applied Sciences in Biomedical and Communication Technologies (ISABEL), 2010 3rd International Symposium on, Nov 2010, pp. $1-5$.

[18] M. A. Poletti, "The application of linearly swept frequency measurements," The Journal of the Acoustical Society of America, vol. 84, no. 2, pp. 599-610, August 1988 .

[19] S. Temel, M. C. Vuran, and R. K. Faller, "A primer on vehicle-to-barrier communications: Effects of roadside barriers, encroachment, and vehicle braking," Department of Computer Science and Engineering, University of Nebraska-Lincoln, Tech. Rep. TR-UNL-CSE-2016-0002, June 2016. [Online]. Available: http://cse-apps.unl.edu/facdb/publications/TR-UNL-CSE-2016-0002.pdf 